Research Paper

\title{
MiR-93 Promotes Tumorigenesis and Metastasis of Non-Small Cell Lung Cancer Cells by Activating the PI3K/Akt Pathway via Inhibition of LKBI/PTEN/CDKNIA
}

\author{
Chunmei Li1 ${ }^{1}$, Jianxin Lyu ${ }^{1 凶}$, Qing H. Meng1,2凶 \\ 1. Key Laboratory of Laboratory Medicine, Ministry of Education of China, Zhejiang Provincial Key Laboratory of Medical Genetics, School of Laboratory \\ Medicine and Life Sciences, Wenzhou Medical University, Wenzhou, Zhejiang 325035, China; \\ 2. Department of Laboratory Medicine, The University of Texas MD Anderson Cancer Center, Houston, TX 77030, USA. \\ $\square$ Corresponding authors: Jianxin Lyu, jxlu313@163.com; or Qing H Meng, qhmeng@mdanderson.org \\ (c) Ivyspring International Publisher. This is an open access article distributed under the terms of the Creative Commons Attribution (CC BY-NC) license \\ (https://creativecommons.org/licenses/by-nc/4.0/). See http://ivyspring.com/terms for full terms and conditions.
}

Received: 2016.10.17; Accepted: 2016.12.23; Published: 2017.03.07

\begin{abstract}
Background: Non-small cell lung cancer (NSCLC) accounts for approximately $85 \%$ of clinical lung cancer cases. MicroRNA-93 (miR-93) is an oncomiR in many types of human cancer, exerting pivotal effects in the development and progression of malignancies, including NSCLC. However, the mechanism underlying miR-93 involvement in NSCLC is unknown. Our purpose was to reveal and explain this mechanism, with the goal of contributing to the development of new diagnostic biomarkers and individualized therapeutic tools.

Methods: The expression of miR-93 was determined in NSCLC cell lines A549, H1975, and H1299. The cells were transfected with control plasmids (Mock group), miR-93 overexpression plasmids (miR-93 Up group), or miR-93 inhibitor plasmids (miR-93 Down group) to generate stable miR-93-overexpressing or-depleted cells. The effects of miR-93 on proliferation, migration, and invasion of these cells were determined. The in vivo effects of miR-93 on tumor metastasis were determined in an NSCLC xenograft mouse model. The molecular mechanisms underlying these effects were investigated via dual luciferase reporter assay and western blotting.

Results: MiR-93 expression levels were significantly greater in the NSCLC cell lines than in normal lung epithelial cells. Cell proliferation, migration, and invasion were significantly stimulated by miR-93 upregulation (all $P<0.05$ ) and inhibited by miR-93 downregulation. Dual luciferase reporter assay demonstrated that miR-93 directly bound with the 3'-untranslated region of the tumor suppressor gene $L K B I$. Western blotting analysis indicated that miR-93 activated the PI3K/Akt pathway by inhibiting LKB1, PTEN, and p21. Increased expression of miR-93 induced significant hepatic metastasis of lung cancer in the xenograft mouse model.

Conclusion: Overexpression of miR-93 facilitates tumorigenesis and metastasis of NSCLC. These findings provide novel insight into the mechanism of miR-93 involvement in NSCLC, suggesting that miR-93 may serve as a potential therapeutic target.
\end{abstract}

Key words: non-small cell lung cancer, miR-93, LKB1, PI3K/Akt, metastasis.

\section{Introduction}

Lung cancer is the most common cancer and the leading cause of cancer death in the USA and China $[1,2]$. Lung carcinoma is generally classified as either small-cell lung carcinoma (about 20\% of all lung carcinomas) or non-small cell lung carcinoma (NSCLC; about $80 \%$ of all lung carcinomas and about $85 \%$ of clinical lung cancer cases) [3]. Although new molecular targeted therapies have shown promising 
results in some types of cancer, no known targeted therapy can be applied to a large proportion of lung cancer patients. Despite improvements in early diagnosis of lung cancer, most lung cancers are still diagnosed at an advanced stage. Therefore, the identification of novel diagnostic biomarkers or treatment strategies is critical and essential for the control of lung cancer.

Because of their characteristic nature, microRNAs (miRNAs) have potential for development as diagnostic, prognostic, and targeted therapeutic tools [4]. Specific miRNAs, such as miR-21, miR-93, and the miR-17-92 cluster (miR-17, miR-18a, miR-19a, miR-19b-1, miR-20a, and miR-92a-1), have been proven to have important regulatory effects on cell proliferation in a variety of human cancers, including NSCLC [5-10]. MiR-93, which belongs to the miR-106b-25 cluster, is an oncomiR in many types of human cancers [11, 12], including NSCLC [13-18]. The aberrant expression and dysfunction of miR-93 have been associated with tumor progression, metastasis, and poor prognosis in hepatocellular carcinoma [7], lung cancer [15], breast cancer [19], gastric carcinoma [14], and nasopharyngeal carcinoma [18]; these effects are mediated through downregulation of various tumor suppressor genes, such as PTEN, CDKN1A, NEDD4L, SMAD7, PDCD4, and TGF $\beta R 2[7,14,15,20]$. MiR-93 promotes cancer progression by targeting PTEN in osteosarcoma, ovarian cancer, and glioma [21-23]. MiR-93 expression has been shown to be increased in tissues and plasma of NSCLC patients and to be a biomarker for early-stage NSCLC [9, 24]. The mechanism of miR-93 involvement in NSCLC is unknown, however. Our purpose was to define the mechanism of miR-93 involvement in NSCLC and thus provide a novel potential strategy for molecular targeted therapy for NSCLC.

\section{Materials and Methods}

\section{Cell lines and cell culture}

NSCLC cell lines A549, NCI-H1975, and NCI-H1299 were purchased from the cell bank of the Chinese Academy of Sciences (Shanghai, China). Each cell line's genetic background is shown in Table 1. The normal lung epithelial cell line BEAS-2B was originally purchased from ATCC (Manassas, VA, USA) and was a gift from Mr. Yilin, Pang, a researcher in our laboratory. All cell lines were authenticated by the cell bank of the Chinese Academy of Sciences or ATCC by using the DNA-fingerprinting method. Cells were cultured in Dulbecco minimal essential medium (DMEM) supplemented with 10\% (v/v) heat-inactivated fetal bovine serum (FBS) (Natocor,
Cordoba, Argentina) and antibiotics (100 U/mL penicillin and $100 \mu \mathrm{g} / \mathrm{mL}$ streptomycin) in a humidified atmosphere of $5 \% \mathrm{CO}_{2}$ at $37^{\circ} \mathrm{C}$.

Table 1. Genetic background of NSCLC cell lines

\begin{tabular}{lllll}
\hline $\begin{array}{l}\text { NSCLC cell } \\
\text { line }\end{array}$ & \multicolumn{2}{l}{ Histology } & PTEN status & \multicolumn{2}{l}{ KRAS/LKB1 status p53 status } \\
\hline NCI-H1299 & AD & wt & wt & loss \\
A549 & AD & wt & c,35G>A;p,G12S10 & wt \\
& & & 9C>T; Q37Ter & \\
NCI-H1975 & AD & L858R and T790M & wt & R273H \\
\hline
\end{tabular}

Note: NSCLC, non-small cell lung carcinoma; AD, adenocarcinoma; wt, wild type.

\section{MiR-93 expression in NSCLC and BEAS-2B cell lines}

MiRNAs were extracted from cells by using the method described in our previous study [5]. Taqman probes (miR-93, U6) were acquired from the Taqman miRNA assay (Life Technologies, Carlsbad, CA, USA). Subsequent detection by qPCR was performed using the TaqMan PCR Master Mix (TAKARA, Dalian, China) with the StepOne Plus Real Time PCR Detection System (Life Technologies). MiR-93 expression was quantified by the relative quantification method with U6 as the reference gene and was verified by comparing with the control cells.

\section{MiR-93 transfection in NSCLC cell lines}

NSCLC cells $\left(2 \times 10^{5}\right)$ were transfected with $1 \mu \mathrm{g}$ of plasmids expressing miR-93 inhibitor sponge (miR-93 Down group), miR-93 (miR-93 Up group), or control miRNA (Mock group) (Genepharma, Shanghai, China). Transfection into subconfluent cells was carried out according to the manufacturer's instructions using Lipofectamine3000 for $48 \mathrm{~h}$ (Invitrogen-Life Technologies). Proliferation, migration, and invasion were determined in these plasmid-transfected cells.

NSCLC cells were transfected with lentivirus containing the miR-93 inhibitor (miR-93 Down group) or control sequence (Mock group) (Genechem, Shanghai, China). Stable miR-93-deficient or control cell lines were obtained after 10 days of incubation in complete DMEM with puromycin $(5 \mu \mathrm{M})$. We pooled the successful clones with green fluorescence for the xenograft tumor mouse model experiments.

\section{NSCLC cell proliferation, colony formation, migration, and invasion}

Cell proliferation was quantified by measurement of absorbance of water-soluble tetrazolium salt at $450 \mathrm{~nm}$, using the Cell Counter Kit-8 (Dojindo, Kumamoto, Japan) as described previously. Target cells (500 cells) were seeded into 6-well plates and incubated with DMEM with 10\% 
FBS for 15 days. Cells were then fixed with $4 \%$ formaldehyde for $20 \mathrm{~min}$, stained with crystal violet for $20 \mathrm{~min}$ at ambient temperature, and photographed. The colony-formation capacity of each group of cells was assessed by counting the colonies formed.

Cell migration was assessed by a transwell migration assay. In brief, transfected cells were resuspended in serum-free DMEM and seeded $\left(2.5 \times 10^{4}\right.$ cells per well) into a 24 -well transwell plate with an $8-\mu \mathrm{m}$ pore membrane insert (Corning, Corning, NY, USA). DMEM with 10\% FBS was placed in the lower chambers as a chemoattractant, and cells were incubated for $24 \mathrm{~h}$. Cells that penetrated the membrane were fixed with $4 \%$ paraformaldehyde for $20 \mathrm{~min}$, stained with crystal violet for $20 \mathrm{~min}$ at ambient temperature, photographed under a microscope (Nikon, Tokyo, Japan), and counted by ImagePro Plus 6.0 software.

For the cell invasion assay, transfected cells were resuspended in serum-free DMEM and seeded $\left(2.5 \times 10^{4}\right.$ cells per well) into a 24 -well transwell plate with an $8-\mu \mathrm{m}$ pore membrane insert, each well coated with matrigel (1:10 diluted with DMEM; BD Biosciences, Franklin Lakes, NJ, USA). DMEM with $10 \%$ FBS was placed in the lower chambers as a chemoattractant, and cells were incubated for $24 \mathrm{~h}$. Cells that penetrated the membrane were fixed with $4 \%$ paraformaldehyde for $20 \mathrm{~min}$, stained with crystal violet for $20 \mathrm{~min}$ at ambient temperature, photographed under a microscope (Nikon, Tokyo, Japan), and counted.

\section{Western blotting}

Cell lysates were prepared for western blotting in Protein Lysis Buffer (Beyotime, Shanghai, China) according to the manufacturer's directions. Protein concentrations were determined by the BCA Assay (Beyotime). An aliquot of $20 \mu \mathrm{g}$ of denatured protein from each sample was subjected to $10 \%$ sodium dodecyl sulfate-polyacrylamide gel electrophoresis and transferred to a nitrocellulose membrane, followed by incubation at ambient temperature with $3 \%$ skimmed milk for $1 \mathrm{~h}$, and then with primary antibody for $1 \mathrm{~h}$ (1:1000 10000 dilution, Abcam, Cambridge, MA, USA), then with horseradish peroxidase-conjugated secondary antibody (1:3000 dilution; Beyotime) for $1 \mathrm{~h}$. The blots were then incubated with enhanced chemiluminescence solution for $1 \mathrm{~min}$. The signals were detected by the ChemiDoc XRS+ Chemiluminescence imaging system (Bio-Rad, Hercules, CA, USA) and quantified by densitometry using ImageJ software. $\beta$-actin was used as an endogenous control to normalize expression data.

\section{Luminescent reporter gene transfection and luciferase assays}

MiRNA targets were predicted by using the TargetScan database (http://www.targetscan.org/). For the luciferase activity assay, 200-nucleotide sequences (the complete predicted miR-93 target site or the mutant miR-93 target site) of the LKB1 3'-untranslated region (UTR) were inserted downstream of the Renilla luciferase gene in a Renilla/firefly luciferase reporter plasmid, psiCHECK-2 (GenePharma). The psiCHECK-2 plasmids and miR-93 mimic were synthesized by GenePharma. 293T cells were transfected with $0.5 \mu \mathrm{g}$ of reporter plasmids containing the miR-93 target site or the mutant miR-93 target site, or empty plasmid, followed by dealing with miR-93 mimic. Twenty-four hours after transfection, Renilla/firefly luciferase activity was measured by a dual luciferase reporter assay (Promega, Fitchburg, WI, USA) in an automatic microplate reader (Thermo Fisher Scientific, Waltham, MA, USA).

\section{Xenograft tumor model in mice}

Twenty male BALB/c nu/nu nude mice (aged 3 weeks, 17-22 g) were purchased from the Shanghai Medical Experimental Animal Care Commission (Shanghai, China). The mice were housed and manipulated in specific pathogen-free conditions using a laminar airflow rack with continuous access to sterilized food and autoclaved water and controlled light/dark cycle, temperature, and humidity. All experiments were performed in accordance with the Guide for The Care and Use of Laboratory Animals of the U.S. National Institutes of Health. The protocol was approved by the Laboratory Animal Ethics Committee of Wenzhou Medical University, Wenzhou, China (Permit Number: wydw2015-0120).

Experiments commenced after 1 week of mouse acclimatization. The mice were divided randomly into two groups (Mock group and miR-93 Down group). Each mouse was injected with A549 cells transfected with miR-93 inhibitor (miR-93 Down) or control (Mock) miRNA $\left(4 \times 10^{6}\right.$ cells per mouse in $50 \mu \mathrm{L}$ of serum-free medium mixed with $50 \mu \mathrm{L}$ matrigel) into the right middle lobe of the lung. On day 35 , five mice of each group were anesthetized with chloral hydrate and killed by decapitation, and their lungs and liver with tumors were removed and photographed. The rest five mice of each group were raised until they dead naturally.

\section{Statistical analysis}

The one-way ANOVA and Student $t$-tests were used, with the Welch correction for variables, to assess the significance of differences between groups. All 
statistical analyses used GraphPad 6.0 software. A P-value $<0.05$ was considered statistically significant. For the in vitro studies, data (mean $\pm S D ; n=3$ ) and images shown are representative of one experiment. Similar results were obtained in three independent experiments.

\section{Results}

\section{MiR-93 is overexpressed in NSCLC cell lines and promotes their proliferation}

MiR-93 expression in A549, H1975, and H1299 cells was 2.57-, 3.17-, and 3.72-fold higher, respectively, than in BEAS-2B cells (all $P<0.001$, Figure 1A). The proliferation of A549 cells in the miR-93 Up group was significantly greater than that of the Mock group after culture for $48 \mathrm{~h}(P<0.01$, Figure 1B). The proliferation of the H1975 and H1299 cells in the miR-93 Up groups were significantly greater than that in the Mock groups after culture for $24 \mathrm{~h}$ (all $P<0.05$, Figures 1C-D). The proliferation of A549 cells in the miR-93 Down group was significantly attenuated compared with the Mock group after culture for $48 \mathrm{~h}(P<0.01$, Figure $1 \mathrm{~B})$. The proliferation of H1975 and H1299 cells in miR-93 Down groups was attenuated compared with the Mock groups after culture for $72 \mathrm{~h}$ (all $P<0.01$, Figures 1C-D).

Similar results were observed in the colony-formation assay. The number of colonies formed by A549 cells in the Mock group, the miR-93 Up group, and the miR-93 Down group were $198 \pm 18$, $266 \pm 25$, and 123 \pm 23 , respectively (Figures 2A-B).
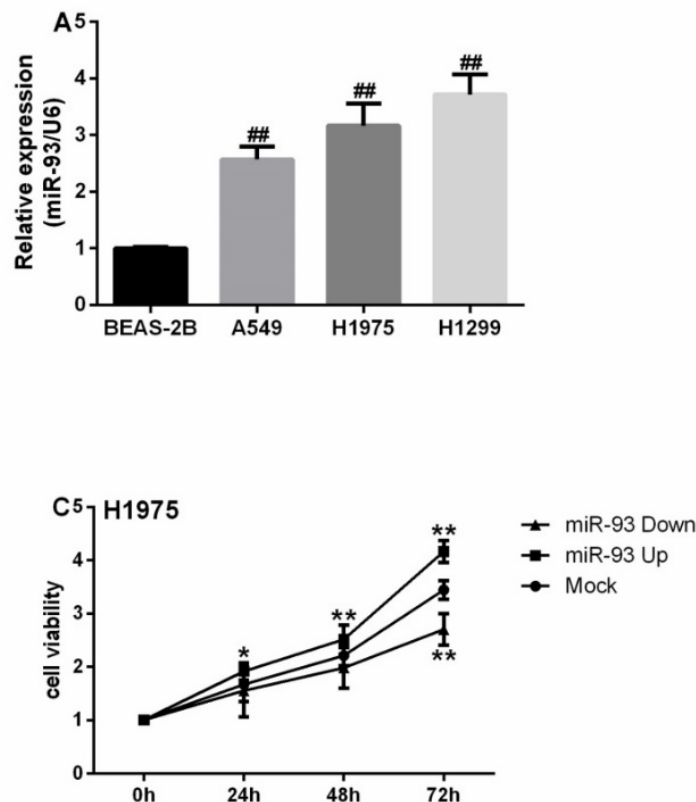

Compared with the Mock group, the colony-forming capacity of the miR-93 Up group was significantly enhanced $(P<0.05)$ and that of the miR-93 Down group was significantly attenuated $(P<0.05)$. The colonies formed by H1299 cells in the Mock group, the miR-93 Up group, and the miR-93 Down group numbered 57 $\pm 5,86 \pm 11$, and $24 \pm 10$, respectively (Figures 2C-D). Compared with the Mock group, the colony-forming capacity of the miR-93 Up group was enhanced $(P<0.05)$, and that of the miR-93 Down group was attenuated $(P<0.01)$.

\section{MiR-93 overexpression promotes migration and invasion of NSCLC cells}

The migration of NSCLC cells was promoted by overexpression of miR-93 and attenuated by inhibition of miR-93. Compared with Mock group, A549 cells in the miR-93 Up group had greater penetration ( $130 \pm 11$ vs $97 \pm 9, P<0.05)$, whereas those in the miR-93 Down group had reduced penetration (58 \pm 16 vs 97 $\pm 9, P<0.05$; Figures $3 A-B)$. In H1975 cells, the miR-93 Up group also had significantly greater penetration than the Mock group (188 \pm 47 vs $100 \pm 7$, $P<0.05)$; the miR-93 Down group had reduced penetration, but the difference was not significant (61 \pm 5 vs $100 \pm 7, P>0.05$; Figures 3C-D). In H1299 cells, the miR-93 Up group had significantly greater penetration than the Mock group $(109 \pm 11$ vs $81 \pm 3$, $P<0.01)$, and the miR-93 Down group had significantly less penetration $(62 \pm 6$ vs $81 \pm 3, P<0.05$; Figures 3E-F).
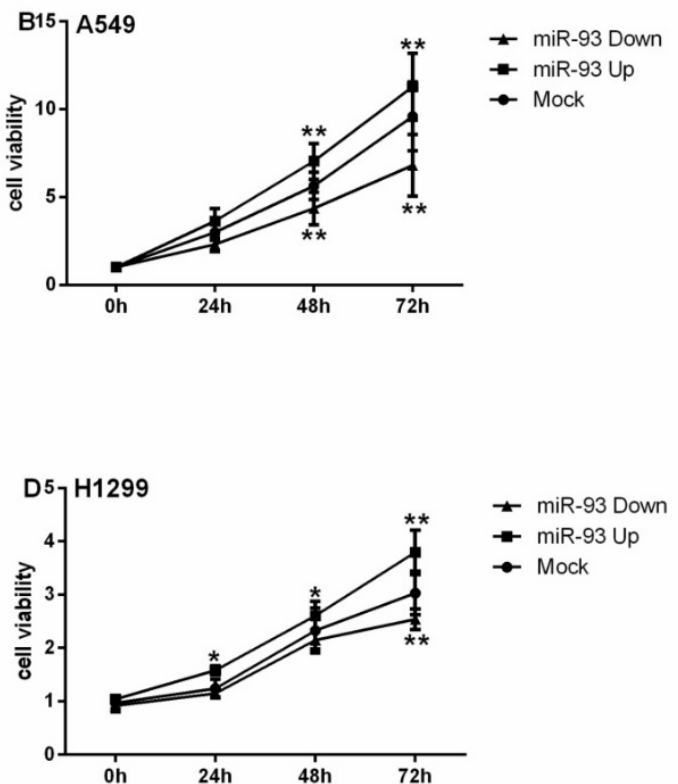

Figure 1. miR-93 is overexpressed in NSCLC cell lines and promotes NSCLC cell proliferation in vitro. (A) miR-93 levels were determined in NSCLC cell lines (A549, H1975, H1299) and normal lung epithelial cells (BEAS-2B). (B-D) Cells were transfected with miR-93 mimic (miR-93 Up), miR-93 inhibitor sponge (miR-93 Down), or control miRNA (Mock) for $72 \mathrm{~h}$ and subjected to a proliferation assay. (B) A549 cells. (C) H1975 cells. (D) H1299 cells. \#\#, $P<0.01$, compared with BEAS-2B. $* P<0.05$, $* * P<0.01$, compared with Mock. 

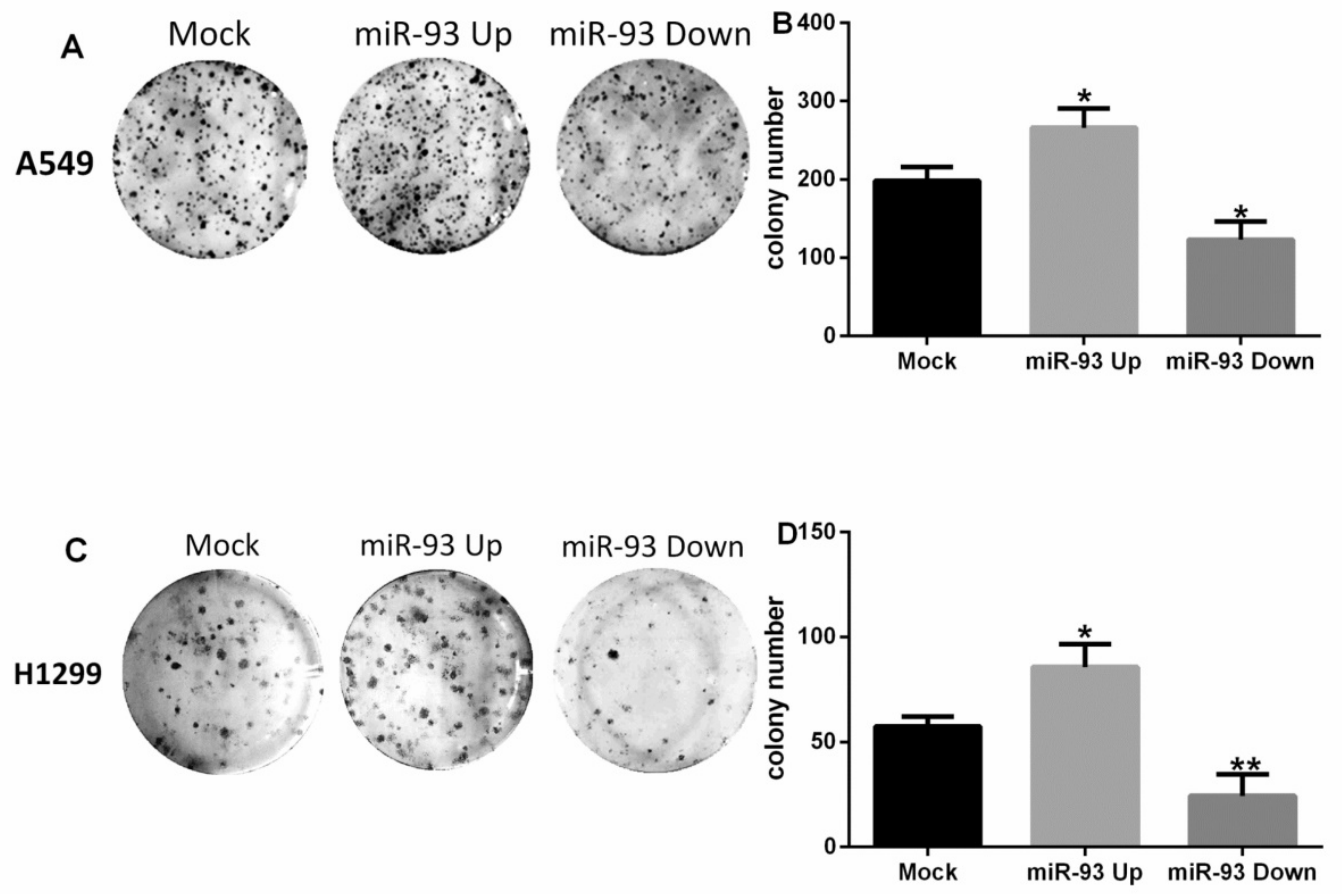

Figure 2. miR-93 promotes colony formation by NSCLC cells in vitro. Cells were transfected with miR-93 mimic (miR-93 UP), miR-93 inhibitor sponge (miR-93 Down), or control miRNA (Mock) and incubated in 6-well culture plates. Colonies were counted after 15 days. (A-B) A549 cells. (C-D) H1299 cells. $* P<0.05$, $* * P<0.01$, compared with Mock.

Similar results were observed in the transwell invasion assay. Invasion was enhanced in NSCLC cells overexpressing miR-93 and inhibited in NSCLC cells in which miR-93 was downregulated. In A549 cells, invasion was significantly greater in the miR-93 Up group than in the Mock group (101 \pm 17 vs $62 \pm 13$, $P<0.05)$, while invasion was decreased in the miR-93 Down group ( $32 \pm 5$ vs $62 \pm 13, P<0.05$; Figures $4 A-B)$. In H1975 cells, invasion also was greater in the miR-93 Up group than in the Mock group ( $86 \pm 14$ vs $60 \pm 3$, $P<0.05)$ and decreased in the miR-93 Down group ( $39 \pm 3$ vs $60 \pm 3, P<0.05$; Figures $4 C-D)$. In H1299 cells, similarly, invasion was significantly greater in the miR-93 Up group than in the Mock group $(86 \pm 14$ vs $60 \pm 3, P<0.01)$ and decreased in the miR-93 Down group ( $39 \pm 3$ vs $60 \pm 3, P<0.05$; Figures $4 \mathrm{E}-\mathrm{F}$ ).

\section{MiR-93 activates the PI3K-Akt pathway by targeting LKBI/PTEN/CDKNIA}

Analysis in silico indicated that LKB1, PTEN, and CDKN1A were candidate targets of miR-93. Katsuya et al. showed, using a dual luciferase assay, that PTEN and CDKN1A are direct targets of miR-93 [7]. Results of our dual luciferase assay showed that LKB1 is a novel candidate target of miR-93 with a 7 mer binding site (Figure 5A). To demonstrate direct binding of miR-93 to this gene, we co-transfected plasmids containing miR-93 mimic, control miRNA, or control together with a vector expressing LKB1 wild-type 3'-UTR, LKB1 mutant 3'-UTR, or empty vector into 293T cells and analyzed their luciferase activity. 293T cells transfected with miR-93 mimic had $64 \%$ lower luciferase activity than parental 293T cells, 293T cells transfected with the control plasmid, or 293T cells transfected with the plasmid expressing LKB1 mutant 3'-UTR (all $P<0.01$; Figure $5 B$ ), with no obvious difference among these three groups. This suggested that LKB1 is a target of miR-93.

In H1299 cells, the levels of LKB1, PTEN, and p21 proteins were lower in the miR-93 Up group than in the Mock group (all $P<0.01)$ and increased in the miR-93 Down group $(P<0.01, P<0.05, P<0.05$; Figures 6A-B). Conversely, the phosphorylation of Akt was increased in the miR-93 Up group $(P<0.01)$ and decreased in the miR-93 Down group $(P<0.01)$. Because of the mutation of LKB1 in A549 cells, we determined the levels of PTEN/p53/p21, phosphorylated Akt (p-Akt), and all-Akt in A549 cells. In A549 cells, similarly, the expression of PTEN, p53, and p21 was lower in the miR-93 Up group than in the Mock group $(P<0.01, P>0.05, P<0.01)$ and increased in the miR-93 Down group $(P<0.01, P<0.05, P<0.05$; Figures 6C-D). Again, the phosphorylation of Akt was increased in the miR-93 Up group $(P<0.01)$ and decreased in the miR-93 Down group $(P<0.01)$. 

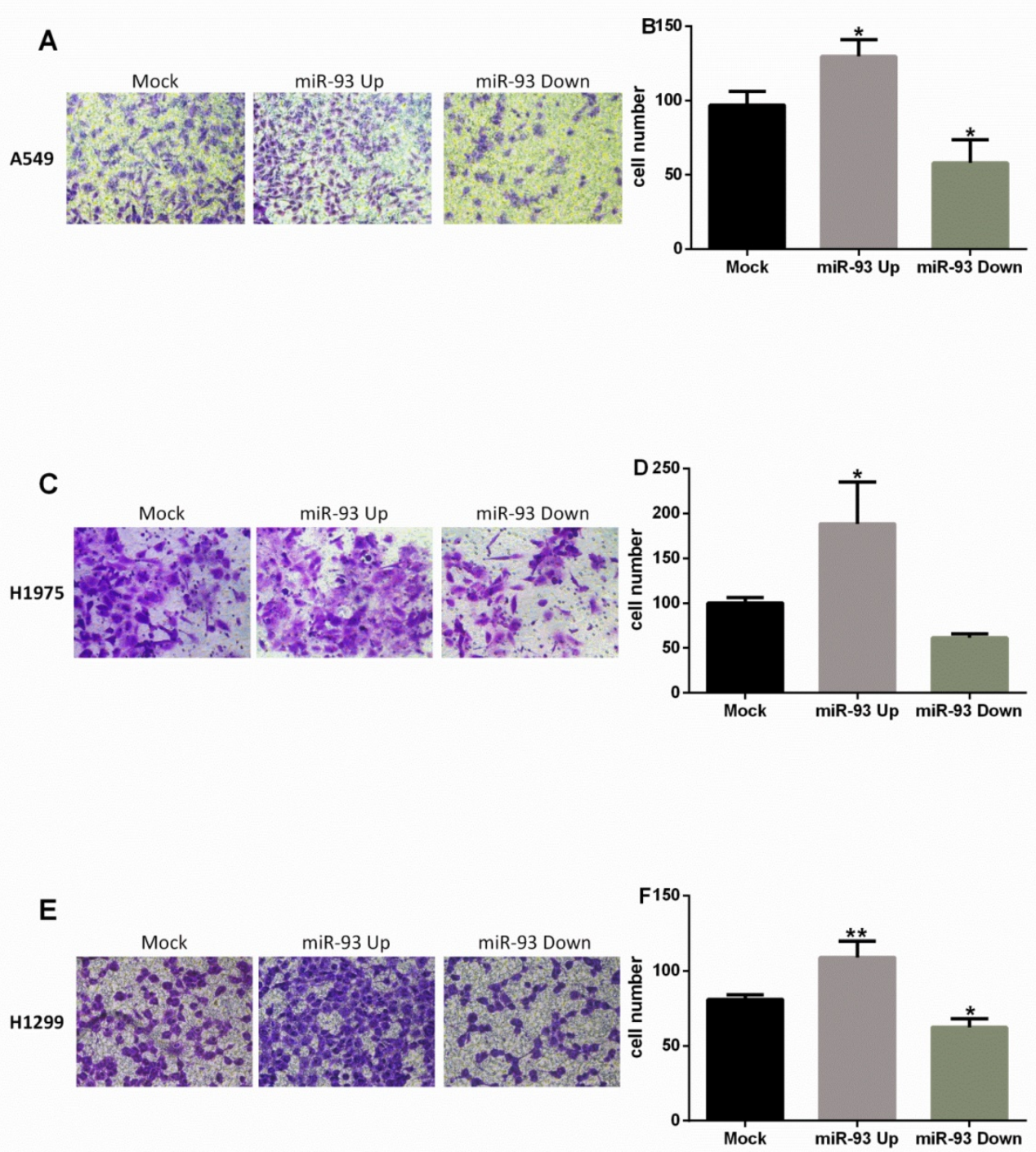

Figure 3. miR-93 promotes NSCLC cell migration in vitro. Cell migration was assessed by determining the number of cells that migrated through the transwell chamber. Cells were transfected with miR-93 mimic (miR-93 Up), miR-93 inhibitor sponge (miR-93 Down), or control miRNA (Mock) and placed in the upper chambers. Cells that penetrated to the lower chambers were counted. (A-B) A549 cells. (C-D) H1975 cells. (E-F) H1299 cells. $* P<0.05$, $* * P<0.01$, compared with Mock.

\section{Downregulation of miR-93 inhibits metastasis and prolongs the survival time in a NSCLC xenograft mouse model}

The tumors that developed from the miR-93 Down A549 cells were associated with significantly lower rates of pleural dissemination and hepatic metastasis than those that developed from controls (Figure 7A). The rate of tumorigenesis was not significantly different between the Mock group and the miR-93 Down group (100\% vs 100\%). The miR-93 Down group had a lower rate of pleural dissemination than the Mock group ( $80 \%$ vs $100 \%$, $P<0.05)$ and no hepatic metastasis (0 vs $80 \%, P<0.01$ ). The survival time of miR-93 Down group was significantly longer than that of Mock group $(P=0.039$, Figure $7 \mathrm{~B})$, with the median survival time was 58 days vs 53 days.

\section{Discussion}

With this study, we demonstrated that miR-93 promotes lung cancer proliferation and metastasis both in vitro and in vivo and identified $L K B 1$ as a novel target of miR-93 in NSCLC. We further demonstrated that miR-93 inhibits LKB1, PTEN, and CDKN1A, thereby activating proliferation and metastases through the PI3K/Akt pathway in NSCLC. Previous studies have shown that miR-93 promotes proliferation in liver cancer by directly targeting PTEN and CDKN1A [7]. Activation of miR-93 not only blocked LKB1, PTEN, and p21 directly but also blocked PTEN and p21 indirectly by blocking LKB1, because LKB1 is known to induce the expression of PTEN, p53, and p21 [25]. When LKB1, PTEN, and p21 were blocked by miR-93, the PI3K/Akt pathway was activated to promote proliferation, migration, and invasion (Figure 8). 
A
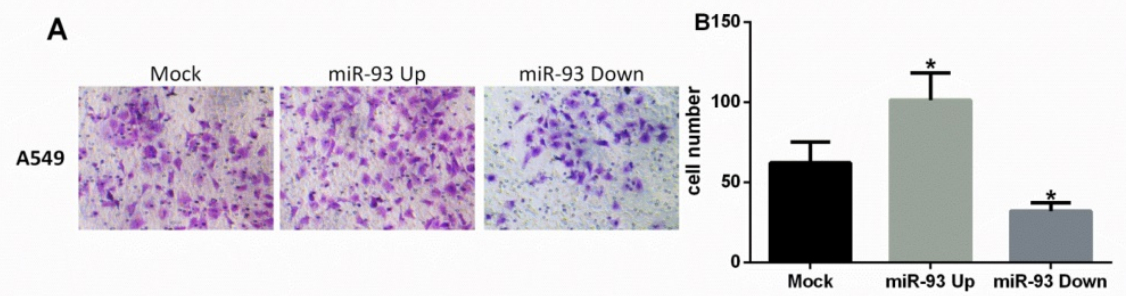

C
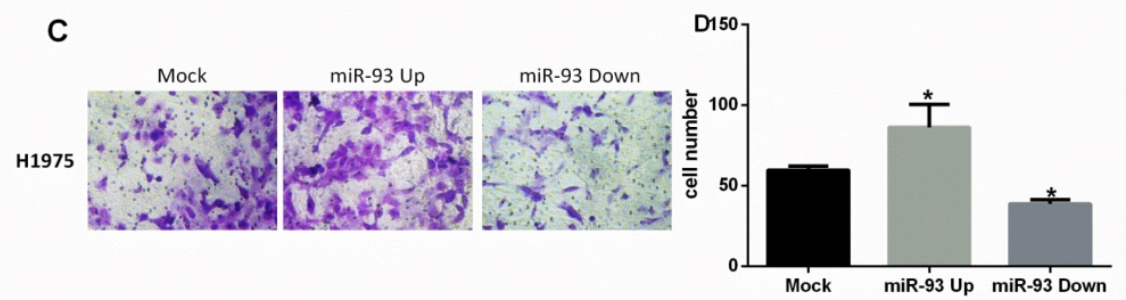

E
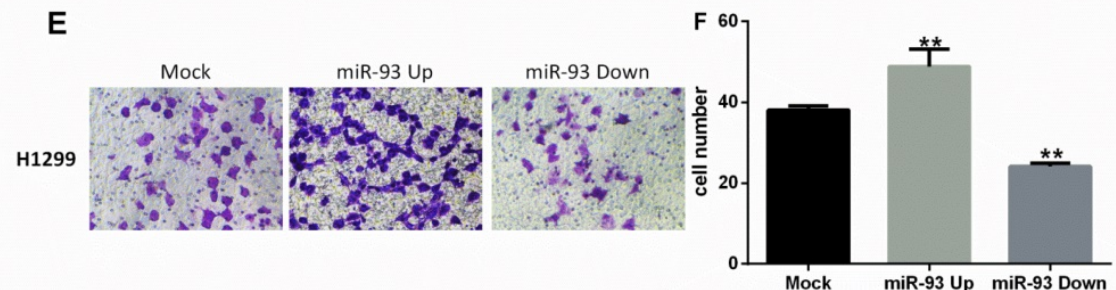

Figure 4. miR-93 promotes NSCLC cell invasion in vitro. Cell invasiveness was assessed by determining the number of cells that migrated through a matrigel coating in a transwell chamber. Cells transfected with miR-93 mimic (miR-93 Up), miR-93 inhibitor sponge (miR-93 Down), or control miRNA (Mock) were placed in the upper transwell chambers and allowed to incubate for $24 \mathrm{~h}$. Cells that penetrated to the lower chambers were counted. (A-B) A549 cells. (C-D) H1975 cells. (E-F) H1299 cells. *P<0.05, $* * P<0.01$, compared with Mock.
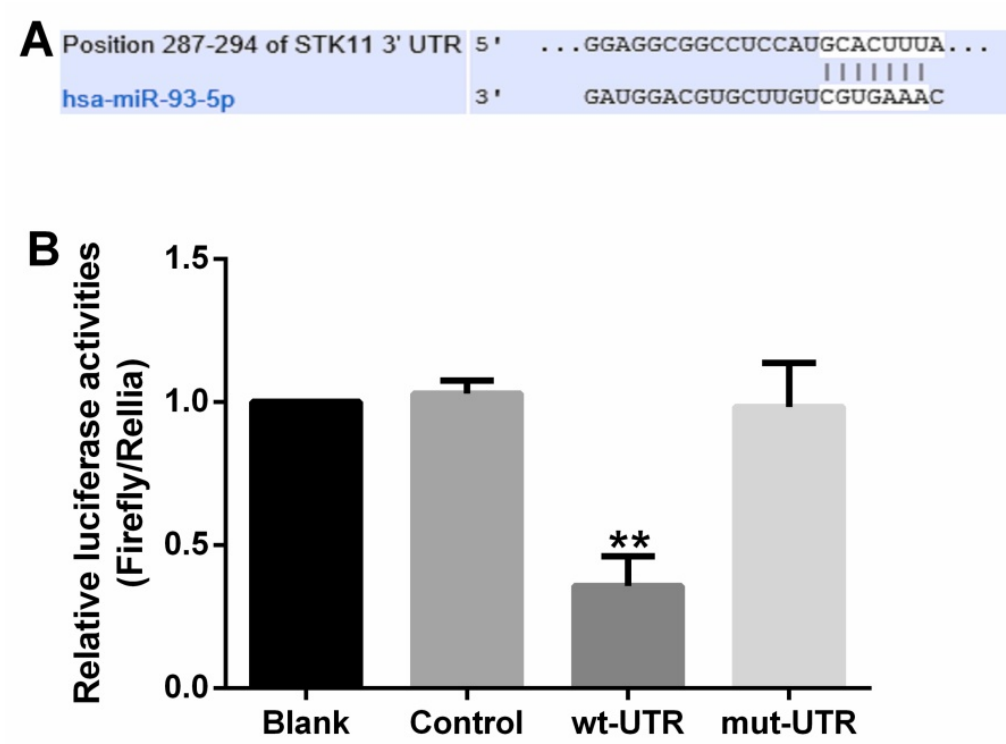

Figure 5. miR-93 directly targets $L K B I$. (A) Sequence alignment of miR-93 and its conserved target site in the $3^{\prime}-U T R$ of $L K B I$. (B) Luciferase activity was measured in $293 T$ cells with a dual luciferase reporter assay. The cells were transfected with plasmids containing the wild-type LKBI $3^{\prime}-U T R$ (wt-3'UTR), mutant LKBI $3^{\prime}-\mathrm{UTR}^{\prime}$ (mut-3'UTR), empty vector (Mock), or nothing (Blank) and co-transfected with miR-93 mimic. Firefly luciferase activity was normalized to Renilla luciferase activity, and the value for the empty vector was used as the control. $* * P<0.01$, compared with all other groups. 


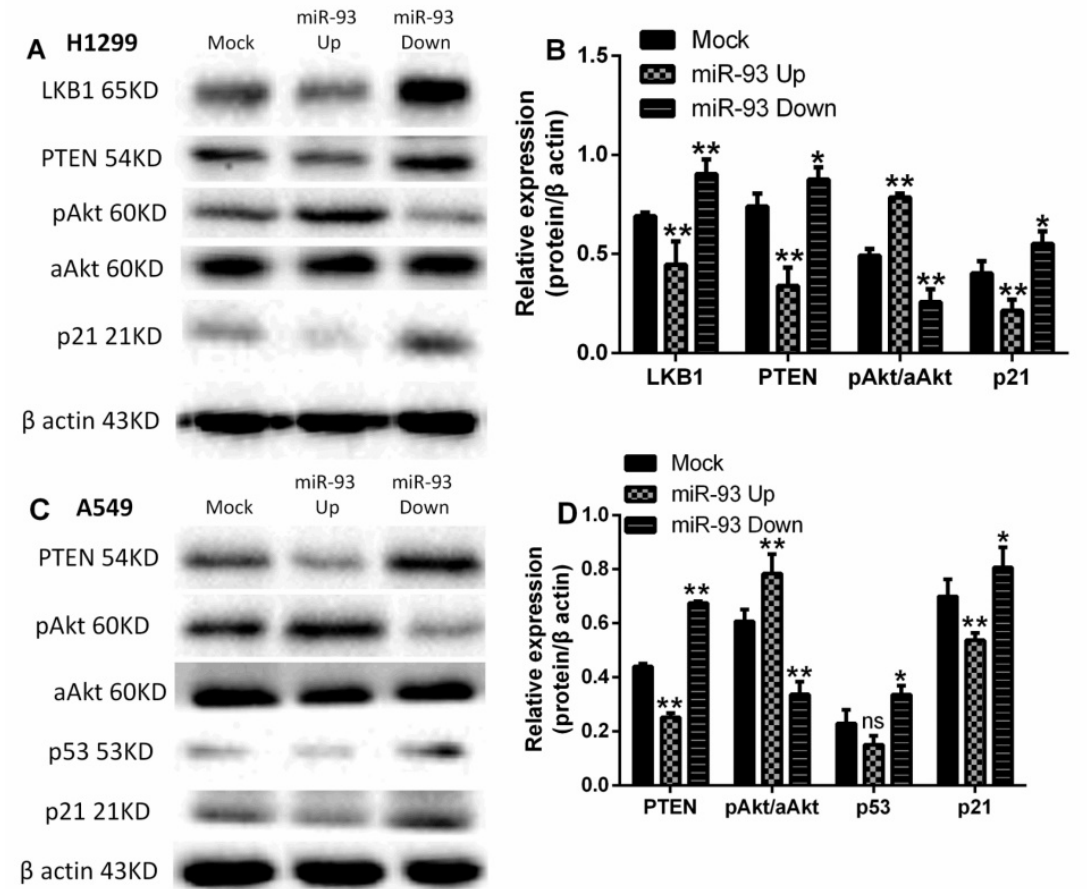

Figure 6. miR-93 regulates expression of LKB1/PTEN/p21 and PI3K/Akt pathway proteins. Levels of the indicated PI3K/Akt pathway proteins were analyzed in (A-B) H1299 and (C-D) A549 cells by western blotting; differences in expression are represented in the bar graphs. Cells were first transfected with miR-93 mimic (miR-93 Up), miR-93 inhibitor sponge (miR-93 Down), or control miRNA (Mock). $* P<0.05$, $* * P<0.01$, compared with Mock.

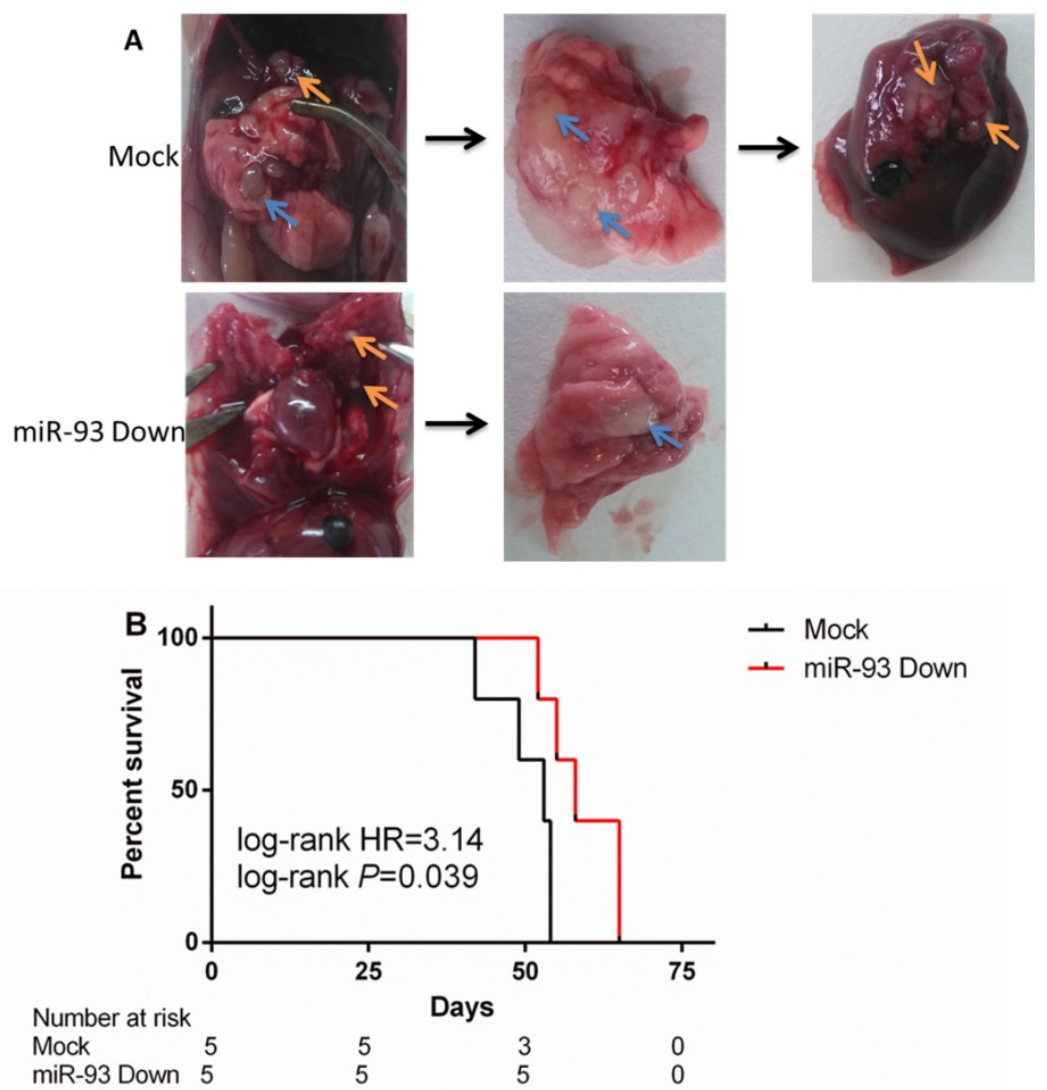

Figure 7. Downregulation of miR-93 inhibits lung cancer hepatic metastasis and prolongs the survival time in vivo. The lung cancer mouse model was constructed by injecting mice with A549 cells transfected with empty lentivirus (Mock) or miR-93 inhibitor lentivirus (miR-93 Down). (A) Five mice of each group were killed on day 35 and their tumors were removed. The blue arrows indicate the primary tumor location. The orange arrows indicate the metastatic tumor location. (B) Survival curve: the rest five mice of each group were raised until dead naturally. 


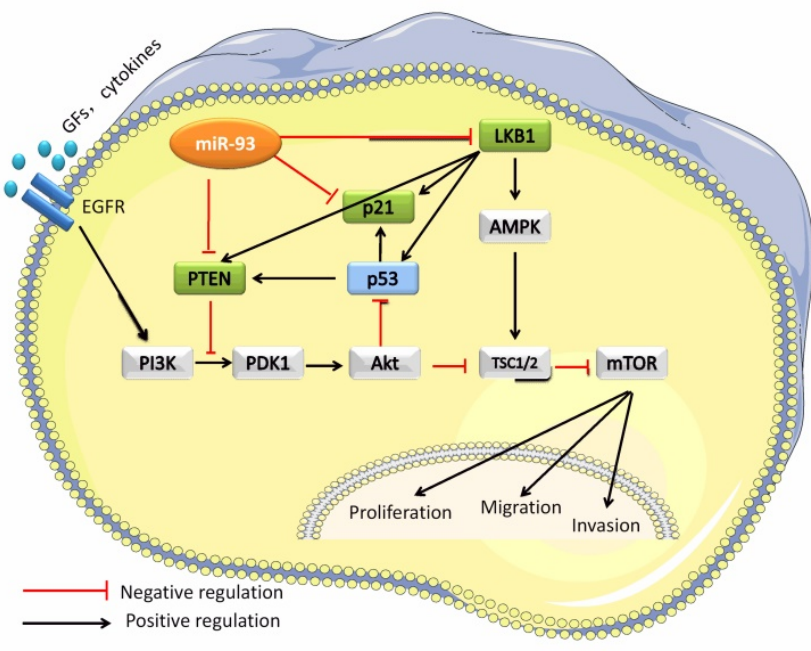

Figure 8. MiR-93 activates the PI3K/Akt pathway by targeting LKB1/PTEN/p21. This schematic figure represents the mechanism whereby miR-93 activates the PI3K/Akt pathway. MiR-93 binds directly binds to the $3^{\prime}-$ UTRs of $L B K I, P T E N$, and CDKNIA.

Our results indicate that the proliferation, migration, and invasion of A549, H1975, and H1299 NSCLC cells were markedly promoted by overexpression of miR-93 and abolished by downregulation of miR-93 in vitro. The hepatic metastasis of A549 tumors in a nude mouse model was significantly inhibited by miR-93 downregulation and the survival time was prolonged. Even though the three cell lines have different genetic backgrounds, the functional changes mediated by miR-93 expression were the same in all three. We speculate that the potential mechanism of miR-93 involvement in NSCLC is based on a multiple target effect.

LKB1, also known as STK11, is a tumor suppressor gene located in chromosome 19p13 and on translation takes on a type kind of serine-threonine kinase function as an inhibitor of mTOR [26]. LKB1 is involved in many biological processes, such as cell cycle control, chromatin remodeling, cell polarity, and energy metabolism. LKB1 mutation is common in Peutz-Jeghers syndrome [27]. In lung cancer, LKB1 is truncated and deactivated via somatic mutation or deletion of $L K B 1$ [28]. The incidence of this is $11 \%-30 \%$ in lung adenocarcinoma [29]. In NSCLC, LKB1 is somatically inactivated in $25 \%$ to $30 \%$ of cases, often concurrently with activating KRAS mutations [30]. Therefore, LKB1 mutation is the third most common mutation in lung adenocarcinoma, after TP53 and KRAS.

Because of the induction of PTEN, p53, and p21 proteins by LKB1 $[25,31,32]$, loss of LKB1 function activates the PI3K/Akt/mTOR pathway to promote proliferation and metastasis [33]. PI3K is the center of a variety of signaling pathways, and it has important off-target effects. Because of the many molecular changes in NSCLC, such as amplification and mutation of PIK3CA, PTEN, and AKT, loss of LKB1, and KRAS mutation, targeting the PI3K/Akt pathway is a rational and promising therapeutic approach in this cancer. Mk-2206, a type of oral protease inhibitor, was combined with Akt inactivation to inhibit NSCLC progression in clinical trials [34].

Our results indicate that miR-93 activates the PI3K/Akt pathway by inhibiting LKB1, PTEN, and p21. We conclude that miR-93 is not only a potential diagnostic biomarker for NSCLC but also a candidate target for treatment. A number of clinical trials using miRNA profiling for patient prognosis and clinical response are currently underway [35-37]. To complete transition of miRNA research from basic to bedside, however, for application of miRNAs as novel biomarkers and treatment targets, several obstacles remain.

\section{Conclusion}

Consistent with the previous studies, we have confirmed that miR-93 is an oncomiR in NSCLC. Moreover, we demonstrate that miR-93 is not only inducing proliferation, migration, and invasion in vitro, but also promoting tumor metastases in vivo, in NSCLC. We demonstrate for the first time that LKB1 is a target of miR-93 in NSCLC. The tumorigenic activity of miR-93 is mediated by activation of the oncogenic PI3K/Akt pathway through inhibition of LKB1, PTEN, and p21 expression. We speculate that miR-93 could be a promising therapeutic target in NSCLC.

\section{Abbreviations}

Akt, protein kinase $\mathrm{B}$; CDKN1A, cyclin-dependent kinase inhibitor 1A; FBS, fetal bovine serum; LKB1, liver kinase B1; miR-93, microRNA-93; mTOR, mammalian target of rapamycin; NSCLC, non-small cell lung cancer; PI3K, phosphatidyl inositol 3-kinase; PTEN, phosphatase and tensin homolog; RT-PCR, real-time quantitative polymerase chain reaction.

\section{Acknowledgements}

We thank Huaibin Zhou, Jindan Wang, Binjiao Zheng, Danli Xie, and Chaowei Wen for technical assistance.

\section{Funding}

This work was supported by grants from the National Natural Science Foundation of China (81170257). This study was also partially supported by the MD Anderson Cancer Center Startup Fund. The funders had no role in study design, data collection and analysis, decision to publish, or preparation of the manuscript. 


\section{Authors' contribution}

QHM, JL, and CL conceived and designed the study. CL carried out most of the experiments. CL, JL, and QHM prepared the manuscript. All authors read and approved the final manuscript.

\section{Ethics approval and consent to participate}

All animal procedures and experimental protocols were approved by Laboratory Animal Ethics Committee of Wenzhou Medical University.

\section{Competing Interests}

The authors have declared that no competing interest exists.

\section{References}

1. Chen W, Zheng R, Baade PD, et al. Cancer statistics in China, 2015. CA Cancer Clin. 2016; 2: 115-32.

2. Siegel RL, Miller KD, Jemal A. Cancer statistics, 2016. CA Cancer J Clin. 2016; 1: 7-30.

3. Inamura K, Ishikawa Y. MicroRNA In Lung Cancer: Novel Biomarkers and Potential Tools for Treatment. J Clin Med. 2016; 3:36.

4. Bartel DP. MicroRNAs: genomics, biogenesis, mechanism, and function. Cell. 2004; 2: 281-97.

5. Li C, Zhao L, Chen Y, et al. MicroRNA-21 promotes proliferation, migration, and invasion of colorectal cancer, and tumor growth associated with down-regulation of sec23a expression. BMC Cancer. 2016; 16: 605

6. Guinot A, Oeztuerk-Winder F, Ventura JJ. MiR-17-92/p38a Dysregulation Enhances Wnt Signaling and Selects Lgr6+ Cancer Stem-like Cells during Lung Adenocarcinoma Progression. Cancer Res. 2016; 76: 4012-22.

7. Ohta K, Hoshino H, Wang J, et al. MicroRNA-93 activates c-Met/PI3K/Akt pathway activity in hepatocellular carcinoma by directly inhibiting PTEN and CDKN1A. Oncotarget. 2015; 5: 3211-24.

8. Yang Y, Meng H, Peng Q, et al. Downregulation of microRNA-21 expression restrains non-small cell lung cancer cell proliferation and migration through upregulation of programmed cell death 4 . Cancer Gene Ther. 2015; 1: 23-9.

9. Du L, Zhao Z, Ma X, et al. MiR-93-directed downregulation of DAB2 defines a novel oncogenic pathway in lung cancer. Oncogene. 2014; 34: 4307-15.

10. Mendell JT. MiRiad roles for the miR-17-92 cluster in development and disease. Cell. 2008; 2: 217-22.

11. Murphy BL, Obad S, Bihannic L, et al. Silencing of the miR-17 92 cluster family inhibits medulloblastoma progression. Cancer Res. 2013; 23: 7068-78.

12. Smith AL, Iwanaga R, Drasin DJ, et al. The miR-106b-25 cluster targets Smad7, activates TGF- $\beta$ signaling, and induces EMT and tumor initiating cell characteristics downstream of Six1 in human breast cancer. Oncogene. 2012; 31: 5162-71.

13. Liu J, Wang H, Wang Y, et al. Repression of the miR-93-enhanced sensitivity of bladder carcinoma to chemotherapy involves the regulation of LASS2. Onco Targets Ther. 2016; 9: 1813-22

14. Liang H, Wang F, Chu D, et al. MiR-93 functions as an oncomiR for the downregulation of PDCD4 in gastric carcinoma. Sci Rep. 2016; 6: 23772.

15. Qu MH, Han C, Srivastava AK, et al. MiR-93 promotes TGF- $\beta$-induced epithelial-to-mesenchymal transition through downregulation of NEDD4L in lung cancer cells. Tumour Biol. 2016; 37: 5645-51.

16. Shi J, Jiang $X, Y u Z$, et al. ZNRF3 contributes to the growth of lung carcinoma via inhibiting $\mathrm{Wnt} / \beta$-catenin pathway and is regulated by miR-93. Tumour Biol. 2016; 37: 3051-7.

17. Li C, Ding C, Chen T, et al. Micro ribonucleic acid-93 promotes proliferation and migration of esophageal squamous cell carcinoma by targeting disabled 2 . Thorac Cancer. 2015; 4 : 524-33.

18. Lyu X, Fang W, Cai L, et al. TGF $\beta R 2$ is a major target of miR-93 in nasopharyngeal carcinoma aggressiveness. Mol Cancer. 2014; 13: 51.

19. Fang L, Du WW, Yang W, et al. MiR-93 enhances angiogenesis and metastasis by targeting LATS2. Cell Cycle. 2012; 23: 4352-65.

20. Yeung ML, Yasunaga J, Bennasser $Y$, et al. Roles for microRNAs, miR-93 and miR-130b, and tumor protein 53-induced nuclear protein 1 tumor suppressor in cell growth dysregulation by human T-cell lymphotrophic virus 1 . Cancer Res. 2008; 21: 8976-85.

21. Kawano M, Tanaka K, Itonaga I, et al. MicroRNA-93 promotes cell proliferation via targeting of PTEN in Osteosarcoma cells. J Exp Clin Cancer Res. 2015; 34: 76

22. Chen Q, Qin R, Fang Y, et al. Berberine Sensitizes Human Ovarian Cancer Cells to Cisplatin Through miR-93/PTEN/Akt Signaling Pathway. Cell Physiol Biochem. 2015; 3: 956-65.
23. Jiang L, Wang C, Lei $\mathrm{F}$, et al. MiR-93 promotes cell proliferation in gliomas through activation of PI3K/Akt signaling pathway. Oncotarget. 2015; 10 : 8286-99.

24. Zhu W, He J, Chen D, et al. Expression of miR-29c, miR-93, and miR-429 as potential biomarkers for detection of early stage non-small lung cancer. PLoS One. 2014; 2: e87780.

25. Jimenez AI, Fernandez P, Dominguez $\mathrm{O}$, et al. Growth and molecular profile of lung cancer cells expressing ectopic LKB1: down-regulation of the phosphatidylinositol 3'-phosphate kinase/PTEN pathway. Cancer Res. 2003; 6: $1382-8$.

26. Shaw RJ, Bardeesy N, Manning BD, et al. The LKB1 tumor suppressor negatively regulates mTOR signaling. Cancer Cell. 2004; 1: 91-9.

27. Shorning BY, Clarke AR. Energy sensing and cancer: LKB1 function and lessons learnt from Peutz-Jeghers syndrome. Semin Cell Dev Biol. 2016; 21-9.

28. Ding L, Getz G, Wheeler DA, et al. Somatic mutations affect key pathways in lung adenocarcinoma. Nature. 2008; 7216: 1069-75.

29. Koivunen JP, Kim J, Lee J, et al. Mutations in the LKB1 tumour suppressor are frequently detected in tumours from Caucasian but not Asian lung cancer patients. Br J Cancer. 2008; 2: 245-52.

30. Liu Y, Marks K, Cowley GS, et al. Metabolic and functional genomic studies identify deoxythymidylate kinase as a target in LKB1-mutant lung cancer. Cancer Discov. 2013; 8: 870-9.

31. Hou L, Liu T, Wang J. Isoflurane suppresses the self-renewal of normal mouse neural stem cells in a p53-dependent manner by activating the Lkb1-p53-p21 signalling pathway. Mol Med Rep. 2015; 5: 7412-8.

32. Xu C, Fillmore CM, Koyama S, et al. Loss of Lkb1 and Pten leads to lung squamous cell carcinoma with elevated PD-L1 expression. Cancer Cell. 2014; 5: 590-604

33. Faubert B, Vincent EE, Poffenberger MC, et al. The AMP-activated protein kinase (AMPK) and cancer: many faces of a metabolic regulator. Cancer Lett. 2015; 2(Pt A): 165-70.

34. Hirai H, Sootome H, Nakatsuru $\mathrm{Y}$, et al. MK-2206, an allosteric Akt inhibitor, enhances antitumor efficacy by standard chemotherapeutic agents or molecular targeted drugs in vitro and in vivo. Mol Cancer Ther. 2010; 7: $1956-67$.

35. Feng B, Zhang K, Wang R, et al. Non-small-cell lung cancer and miRNAs: novel biomarkers and promising tools for treatment. Clin Sci (Lond). 2015; 10: 619-34.

36. Lanford RE, Hildebrandt-Eriksen ES, Petri A, et al. Therapeutic silencing of microRNA-122 in primates with chronic hepatitis $C$ virus infection. Science. 2010; 5962: 198-201.

37. Agostini M, Knight RA. MiR-34: from bench to bedside. Oncotarget. 2014; 4: 872-81. 